\title{
Interlanguage: A Case Study of a Korean Learner of Bangla
}

A.K. M. Mazharul Islam

Lecturer, English Language Centre, Department of English, College of Language and Translation, King Khalid

University, Abha, KSA

Corresponding Author: A.K. M. Mazharul Islam, E-mail: amazharul@kku.edu.sa

\author{
ARTICLE INFO \\ Received: December 04, 2018 \\ Accepted: December 25, 2018 \\ Published: January 31, 2019 \\ Volume:2 \\ Issue: 1 \\ DOI: 10.32996/ijllt.2019.2.1.13 \\ KEYWORDS \\ Interlanguage, transitional \\ competence, phonetic, \\ morphological and syntactic \\ perspectives, pedagogical \\ implications
}

\section{1- INTRODUCTION}

\subsection{Foreword}

One of the main challenges facing many countries is how to maintain their identity in the face of globalization and growing multilingualism. There is a case for regulating the status of English but ways need to be found of reinventing national identity around a distinctive mix rather than a single language which is kept pure. (p.116)

From the above statement of Graddol (2006) it is very clear that at this point of civilization monolingualism is rapidly disappearing from the face of the earth. Ellis (1997, p.3) opines that in the time of 'global village' and 'World Wide Web' people around the world are not merely limited to their own speech communities. Hence learning a second (third, fourth ... ) language is not just a pastime rather it has become inevitable. From the second half of the twentieth century a keen interest arose among the linguists in second language acquisition and they focused their studies to know how people acquire a second language. Collection of the samples of Learner language or Interlanguage has proved to provide a valuable insight in this regard. (Figure in Index)

\subsection{Aim of Study}

In this research, first of all, effort has been taken to make the concept of 'interlanguage' and its various features clear. Then, available literature on Interlanguage is displayed and discussed. Characteristically, all the obtainable literature is about learning English as a second language. There is almost no traceable work on Interlanguage where Bangla has been learnt as a second language. To carry out my research I have interviewed four foreigner learners of Bangla of different nationalities employed in different professions in Bangladesh as my random subjects.

Since the main way of investigating L2 acquisition is by collecting and describing samples of learner language, the major focus of my data collection was to trace various features of Interlanguage in the output the learners and to analyze the errors found in their output from phonetic, syntactic and morphological levels. There might be some common hurdles where most learners stumble. Once we become familiar with the errors they make, our knowledge of their lapses may work as guidance for both teaching and learning Bangla as a second language easily. 


\subsection{Research Questions}

One of the first methodological steps in a research is to formulate a research question. By a research question a researcher formally states the aim of his study. It is usually focused, concise and arguable. The research question states clearly what the study will investigate or attempt to prove. It works as a guideline all through the study. The research question is a rational statement that comes from what is known or believed to be true or understood and accepted from available literature of the concerned topic and it leads the investigator to what is unknown and requires validation and proof. An accurate and clearly defined research question saves a lot of beating about the bush and directs the researcher what is to follow first and foremost.

In this study I moved with one central question along with two sub questions. The key question that I had in this investigation was, "what features of interlanguage are found in the output of the foreigner learners of Bangla?" There are various traits of interlanguage and it is supposed that they appear in the interlanguage when any one learns a language. Do they appear in the foreigner learners' performance in case of learning Bangla? I wanted to know the nature of their linguistic deviations. The second question that I posed was, "Are there any special feature in their effort to communicate in Bangla?" Every language and the speakers of that language are unique to some extent. Does Bangla cause some special feature to arise in the output of the learners? Finally, in a shorter range, my quest was, "what may be attributed to those deviations of language which the learners make?" Are the deviations caused by some individual difference factors like motivation and intelligence or by the interference of their mother tongue? These are the questions that spelled out the scope of my activity in the survey and gave a form to my investigation.

\section{2- LITERATURE REVIEW}

\subsection{Introduction}

The term 'interlanguage' was coined by Selinker $(1969,1972)$ to refer to the progressive knowledge of the second-language learners on their way to the target language. A plethora of terms have been used to mean the language leaner's language. Along with 'interlanguage' it is also called 'interlingua' or 'interlingual identifications' (Weinreich, 1953), 'approximative systems' (Nemser 1971) 'transitional competence' (Corder 1971), 'interim grammar', and 'language learner-language' (Corder 1978) by different scholars at different points in time starting from early sixties.

\subsection{The concept of Interlanguage}

Interlanguage is the midway of a second language learner in his journey towards the rules of second language. This body of knowledge is different from both his mother tongue and the target language. At any given time in the continuum, from a point he usually marches forward but he may also become stagnant or may even slide back. Before we look back into the history of interlanguage let us be familiar with the idea as McLaughlin (1987) puts it:

Generally speaking, the term 'interlanguage' means two things: (1) the learner's system at a single point in time and (2) the range of interlocking system that characterize the development of learners over time. The interlanguage is thought to be distinct from both the learner's first language and from the target language. It evolves over time as learners employ various internal strategies to make sense of the input and to control their own output. (p.60)

\subsection{Interlingual Identifications}

In the history of exploration of psychology of second language learning Weinreich $(1953$, p7) is the pioneer to discuss different aspects of interlanguage, though it was not termed so at his time. He calls it 'interlingual identifications'. He opines that, in a language contact situation, such identifications can develop in the phonemes, in the grammar and in the semantics of the concerned languages. Selinker (1972) criticizes that Weinreich did not make clear where these growth take place. According to Selinker a latent psychological structure in human brain must be assumed for those developments to take place and that latent structure is activated when one learner attempts to learn a second language. Lennenberg (1967, pp. 374-379) calls this structure Latent language structure and according to him in that structure there (a) is an already formulated arrangement in the brain, (b) is the biological counterpart to universal grammar, and (c) is transformed by the infant into realized structure of a particular grammar in accordance with certain maturational stages. Selinker's latent language structure is not exactly the same as Lennenberg's.

\subsection{Transitional Competence}

Corder (1967, 1971, 1978) in his various essays speculates somewhat the same phenomenon of 
interlanguage with different terminologies like 'transitional competence', 'idiosyncratic dialect' and 'language-learner language' etc. $\mathrm{He}$ classifies performance 'mistakes' as unsystematic and 'errors' as systematic; errors occur due to the inadequate knowledge of the system of the target language, and they are termed as transitional competence (Corder, 1967, p 166). According to him, errors are indicative of the developmental state of the fact that learning is taking place. They also prove that learners employ strategies and they have a tendency to induce rules. Corder thinks both first and second language learners employ the same strategies. Corder (1967, p.166) states:

I propose therefore as a working hypothesis that some at least of the strategies adopted by the learner of a second language are substantially the same as those by which a first language is acquired. Such a proposal does not imply that the course or sequence of learning is the same in both cases.

Corder opines that studying language-learner language and their errors is very essential. They will help us to know the learner's innate strategies to dictate our practice and determine our syllabus. The progressive knowledge of the learner will lead us to adapt ourselves to their needs rather than to impose on them our perception of their needs.

\subsection{Approximative systems}

Richards (1974, p 29) says 'Nemser's terminology is a little different from Selinker's but it is applied to precisely the same phenomenon. He uses approximative system for interlanguage". Nemser (1971) first classifies the languages in contact situations as the target language $\left(\mathrm{L}_{\mathrm{T}}\right)$, the source language $\left(\mathrm{L}_{\mathrm{S}}\right)$, an approximative system $\left(\mathrm{L}_{\mathrm{a}}\right)$ and $\mathrm{L}_{\mathrm{a}}$ $\ldots \mathrm{n}$ indices refer to systems at successive stages of proficiency. An approximative system, according to him, is the deviant linguistic system actually employed by the learner attempting to utilize the target language. He also says that learner speech at a given time is the patterned product of a linguistic system. $\mathrm{L}_{\mathrm{a}}$, is distinct from $\mathrm{L}_{\mathrm{s}}$ and $\mathrm{L}_{\mathrm{T}}$, and internally structured. He also states that in a given contact situation, the approximative systems of learners at the same stage of proficiency roughly coincide. Regarding the importance of interlanguage study Nemser (1971) summarizes:

$$
\begin{aligned}
& \text { Investigation of such leaner } \\
& \text { systems is crucial to the } \\
& \text { development of contrastive analysis } \\
& \text { theory and to its applications to }
\end{aligned}
$$

language teaching. However, these systems also merit investigation in their own right through their implications for general linguistic theory. (p.62 in Richard 1974)

\subsection{Interim Grammar}

Selinker (1969, 1971, and 1992) provides the most encompassing discussion on this issue. He says that there is a latent language acquisition structure in the brain of language learners. He maintains that interlanguage studies can be done based "on the observable output which results from a learner's attempted production of a TL norm" and to establish relevant data we need 1) utterances in the learner's native language (NL) produced by the learner; 2) IL utterances produced by the learner; and 3) TL utterances produced by native speakers of that TL. When an investigator has these three sets of utterances within a theoretical framework he can begin to study the psycholinguistic processes which establish the knowledge which underlies IL behavior. Selinker (1972) states:

I would like to suggest that there are five central processes (and perhaps some additional minor ones), and that they exist in the latent psychological structure ...... I consider the following to be processes central to second language learning; first, language transfer; second, transfer of training; third, strategies of second language learning; fourth, strategies of second language communication; and fifth, overgeneralization of TL linguistic material. Each of the analyst's predictions as to the shape of IL utterances should be associated with one or more of these, or other, processes. (p35, in Richards 1974)

These five processes in brief are as follows:

1) Language transfer: some items, rules, and subsystems of the interlanguage may result from transfer from the first language. Example: What did he intended to say? (Selinker,1972)

2) Transfer of training: some elements of the interlanguage may result from specific features of the training process used to teach the second language. Selinker here talks about a Serbo-Croatian learner who always 
mixes up the use of English 'he' and 'she', though the learner had the he/she distinction in his mother tongue.

3) Strategies of second language learning: some elements of the interlanguage may result from a specific approach to the martial to be learned. Example: Don't worry I am hearing him.

4) Strategies of second-language communication: some elements of the interlanguage may result from specific ways people learn to communicate with native speakers of the target language.

5) Overgeneralization of the target language linguistic material: some elements of the interlanguage may be the product of overgeneralization of the rules and semantic features of the target language.

Fossilization according to Selinker is the state of affairs that exists when the learner ceases to elaborate the interlanguage in some respect, no matter how long there is exposure, new data, or new teaching. Among the learners there is a tendency of backsliding, that is, producing the errors of early stage of development of second language learning. Selinker and his associates think that IL development is different from first language development and it caused mostly due to language transfer phenomenon. He cites the example of the French speaker who retain the uvular $/ \mathrm{R} /$ in their English interlanguage, English speaker who use English word order in German sentences. He also admits that it may occur due to other factors. It may be caused by language learning strategy. It may so happen that a learner has learnt enough to communicate then he may siege to learn anymore and will tend to avoid the trouble of learning. Selinker (1992) confirms his view of language transfer and fossilization as crucial aspects of interlanguage and lays farther importance on the extensive study of interlanguage to reach insight in the field of SLA.

Selinker et al. (1975) presents a study where he argues that there is definite systematicity in the interlanguage of the learners. $\mathrm{He}$ says in this systematicity there are some strategies involved like language transfer, overgeneralization of target language rules, and simplification. In this way, as Selinker states, interlanguage is the interim grammar which develops different cognitive strategies - for example, transfer, overgeneralization and the correct understanding of the target language.
There are some remarkable tenets of interlanguage. Consulting the prevailing literature and mainly based on Selinker, Ellis (1999) discusses three major features of interlanguage. They are: language-learner language is permeable, dynamic and systematic. Permeability in interlanguage means the rules that constitute the learner's knowledge at any one stage are not conclusive or fixed rather they are amendable. That is developmental IL knowledge is ready to receive modifications. The dynamic feature refers to the constant changing nature of interlanguage knowledge. The learners slowly accommodate new hypotheses about the target language system. A process of "constant revision and extension of rules is a feature of the inherent instability of interlanguage and its built in propensity for change." (Ellis 1999, p50). The systematic feature of IL refers to the fact that there is a rule based nature of the learners' use of L2. That is IL of the learner is a rule governed behavior. The learner does not haphazardly select rules from the store of his interlanguage rules; rather he does this in a systematic way.

Selinker (1975) shows the involvement three main strategies of language transfer, overgeneralization and simplification, under systematicity feature of interlanguage in a study of 10 boys and 10 girls in a French immersion class. The children were taught by a native speaker. The learners could talk among them in French and could understand the teacher but they had no scope to use French outside the class: The study is as below (Table in Index 2):

Three salient views regarding the development of interlanguage dominated the 70s. Selinker and his associates thought the learners develop the rules of the target language through cognitive strategies like simplification, overgeneralization and language transfer. Adjemian argued that it is a rule goverened behavior and can be analyzed linguistically like any other natural language. It is according to her, is a set of grammatical intuitions. A third approach was backed by Tarone (1979, p.65 in McLaughlin1987). He maintained that the interlanguage could be seen as analyzable into a set of styles that are dependent on the context of use. He gave more importance to the context of use and argued the context to be the determinant. In this way, we see the evolution at work in the interlanguage hypothesis from the beginning when it came into being as a protest against morpheme study and contrastive analysis. 


\section{3- DATA COLLECTION}

\subsection{The Study}

One of the most important and crucial episodes of research is data collection. The main purpose of gathering data is to make important decision based on the collected information through various types of analysis. Inaccurate data may ultimately lead to invalid results. There are several ways of collecting data. Topic and area of the research usually determines the means of collecting data. There are various ways like interviews, face to face or over phone or computer assisted, questionnaire, observation, document review and so on. For my data collection I have used face to face and over phone interviews.

\subsection{Participants}

The interviewee chosen was a student at IML, DU, Bangladesh. I shall be using a pseudonym for my respondent namely Mary. She is an adult learner and received training in Bangla for different periods. She was living in entirely in a TL surroundings. She moved mostly among the educated group of people who are able to use English to communicate them. In brief, she got an extended exposure to the target language.

\subsection{Method}

To collect data I depended mainly on semi-formal interviews with a qualitative approach. I could not follow longitudinal observation, though my topic demanded. The tool I have used mostly was a voice recorder. To conduct my interview I have used a 2 GB Sony IC recorder (ICD-ux81) and mobile phone (Symphony s110). Placing the recorder before the interviewee I let them speak. Some time, I had to give a list of question written in English along with Bangla translation which I was going to ask them just few minutes before the session. For eliciting more data I have asked usually descriptive questions like আপনার নিজের গ্রাম সম্পর্কে বলুন (please tell us something about your village/town) to let them talk freely. The interviews lasted for fifteen minutes on the average. I conducted two interviews of the subject and I tried to make the in-between gap of sessions as long as possible. The maximum gap that I could manage was more than a year. When the interviews were over I firstly backed up the recordings in my PC and online, then I transcribed them for analysis. In all cases I have ensured my subject that all the data will be kept confidential and will be used for research purpose only.

\subsection{Limitations of the study 3.4.1 Delimiting the Topic}

Investigation into Interlanguage and its various features require a vast scope of time and length. Interlanguage studies have various dimensions like social, discourse, psycholinguistic and linguistic aspects. Another important thing is Interlanguage researches are by nature longitudinal, but I had to work, virtually, within one year time. Therefore, I had to zoom in my view and be selective. Narrowing down my focus I have anchored on phonetic, morphological and syntactic deviations that the learners make in their effort to produce Bangla language and tried to analyze them.

\subsubsection{Time}

To do this research I had to meet some tough challenges. The first one was the time. I had to feel the time-tightness all through. The time was very limited to pursue a topic like interlanguage. The topic usually demands a prolonged survey. One of the time consuming job in this study was transcribing the interviews in Bangla. The time which should be allowed to find any significant change in the output of the learner could not be given and I had to rush to finish within time.

\subsubsection{Respondents' Unavailability}

Another constraint was to manage foreign learners to interview and to get a schedule from them. In most cases access was restricted. To talk to them I had to go in a roundabout way of being recommended by my teachers otherwise it was not possible to talk to them. Thus respondent's unavailability and unwillingness to sit for an interview posed a considerable threat to the completion of my study.

\subsubsection{Language Problem}

Another barrier that I had to face was the barrier of language to communicate my interviewee. I had to speak in Bangla mostly to check their comprehension. She understood Bangla if spoken slowly, but at times could not understand and I had to keep repeating. She in some occasions also made complaints that I spoke too fast to follow. Sometimes English was the means of rescue but once you start in English the rest of the conversation ensues in English and it was not easy to come back in Bangla again. Some of their utterances of Bangla were so much affected by their first language that I failed to understand them at times. While transcribing it was a practical problem and I had to listen to a note in the recorder dozens of times. Sometime the utterances in response to the questions created very funny situation. Minimal pairs ষরশব অআধা-আদা ’ ডাকা-ঢাকা, ’ বাছুর-ভাসুরচ , 'ধॉাঁা-দাদাচ ধহফ ত্বাঁধ-বাদচ were not easy to handle and created problems. For example: 
: আপনাদের পুকুর আছে? (Do you have pond?)

: কুকুর? হাঁ হাঁ কুকুর অবশ্যই আছে (Oh, certainly, I have dogs in my house.)

\section{4- DATA ANALYSIS}

\subsection{Introduction}

Mary, the Korean learner of Bangla, has been the subject and her output has been full of interlingual identifications. She yielded ample significant data for much analysis. Mary was not just the beginner and she was also not a refined speaker of Bangla. She was left somewhere midway. Her output was full of fillers, gaps, fumbling, hesitation, repetition and overt request for help. Another feature of her speech was that she was continuously using English words. Though she was a good communicator and maintained the sessions well, her speech gave a clear view of her developing knowledge of Bangla phonology, morphology and syntax.

\subsection{Phonetic}

The developing aspect of her knowledge of Bangla phonology was distinct. Mary had problem with the Bangla aspirated sounds like $/ \mathrm{k}^{\mathrm{h}} /, \mathrm{g}^{\mathrm{h}} /, / \mathrm{t}^{\mathrm{h}} /, / \mathrm{b}^{\mathrm{h}} /$ (' $\mathrm{L}, \mathrm{N}$, _, f')in the words like শিকা (শেখা), তাকে (থাকে), বাল (ভাল)। She said ' দক্ষিন কোরিয়ায় তাকে' in response to the question "where does your family stay?" She says " তাকে" instead of “_ $v \neq K$ ”. She uses the sound $/ \mathrm{t} / \mathrm{n}$ ' $\mathrm{Z}$ ' for the sound / $\mathrm{t}^{\mathrm{h}}$ ('“').In Bangla, language is “ভ/ষो". Mostly she calls it "evlv". Here the sound ' $\mathrm{f}$ ' is very often replaced by 'e'. It is the same with the word 'fvj', she calls 'evj'. Again the second' $Z$ ' is replaced

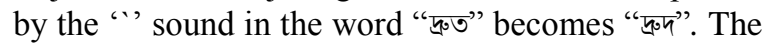
sound "' is taken over by the sound ' $Z$ 'here in her case. The sound /t/ (U) is commonly replaced by' $Z$ ' in the final positions of the words like “जिनতা, গন্" . The velar voiced aspirated $/ \mathrm{g}^{\mathrm{h}} /$, ' $\mathrm{N}$ ' sound is also replaced by unaspirated velar sound /g/' $M$ '. Some of her vowels were also not like the native Bangladeshi. Her $/ \mathrm{o} /(\mathrm{A})$ sound tended to be more round and like 'I' or ' $\mathrm{D}$ ' $/ \mathrm{u} /$ in the words 'হলে, পরে, করে'. Sometimes the final sounds of words were missing. She uttered words like “আজকা (আজকাল), পরিস্কা (পরিস্কার)”where she dropped the final sound. She used “ইংলেজি, ইংলাজি, ইংলিজ, ইংলেশি, ইংলিশ” for the Bangla word “ইংরেজী'. A remarkable feature of her delivery is she also made the right pronunciations occasionally. Along with the ones discussed, there were some other idiosyncratic utterances what suggest that she was yet to develop full competence in respect of phonology of the target language.

\subsection{Morphological}

Mary's performance also indicates the progressive nature of her knowledge of morphology of the target language. For instance, she said, “ কি সম্পর্ক?” in place of " কি সমপর্কে?". Here she fails to add Bangla suffix "G" at the end of the word "সম্পর্ক". In another place she said “ বিভিন্নড়িৎফ ডে পছন্দ করি’ using an extra suffix " $ড ে "$ to the word "word". Regarding Bangla food she said, “.. খাশি মাংস। খাশি মাংস এবং..”. the suffix 'i' is missed here. Elsewhere she said " তাই বাংলা ভাষাও শিখতে চায়'. With the subject I (Avwg) in Bangla the form of the verb should have been " চাই" according to the rules of Bangla grammar. Yet in another place she told, “চাইনিজ লেটার এসেছিলাম," . Here she used “এসেছিলাম" in place "এসেছিল'. Yet in another occasion she said, “চাইনিজ লেটার ব্যবহার করি', where she was supposed to say “ব্যবহার করে'. She also said, “ কোরিয়ান মানুষ.. আমরা-বাষা অনেক পছন্দ করি' while, may be, trying to say, 'কোরিয়ান মানুষ.. নিজেদের ভাষা অনেক পছন্দ করে’ . At one point she said, “তাই শ্যু সালাদেতে খেতে পারি'. She said “ সালাদেতে" without saying “ সালাদ দিত়ে”. She showed a tendency to use "তে" suffix in all cases - " আরেরিকাতে, ব্রিটিশতে, নিউজিল্যান্ডতে, কানাডাতে". Still at another point she said, “ কোরিয়া মানুষ চাতে চিনি খাইনি', it could have been “ কোরিয়াতে মানুষ চাতে চিনি খায়নী”. Again, here the same thing happens, "অনেক আগে কোরিয়া, কোরিয়াতে, কোরিয়ায়ে বাষা না হলে..". In these occasions she was not sure of the bound morpheme she was to use with the word ' কোরিয়া”. In the sentence “ কিন্তু. এবং.. তাহলে তাহলে ফরটিন সেঞ্চুরিস .. ফ.." she was not certain which functional free morpheme would be proper to use. Being asked what languages she knew she replied, " আমি বিভিন্ন বাষা চিনি, সেকেন্ড ল্যাংগুয়েজ, সেকেন্ড ভাষা ইংলাজি, so, and Japanese এবং আরাব, আরাবী আ.. চিনি, French, Spanish চিনি, আরো চাইনিজ চিনি”. Here in this case she was not able to use the word "জানি' in place of "बिनि'. It was may be due to 'language transfer' from her mother tongue Korean or from the knowledge of English language where 'know' is used for knowing a language and also for knowing a person but in Bangla we have two different words " চिनि" and "জানি'. And interesting enough, she was able to use the word “জানি' correctly in her second interview -“... জাপানিজ বাষা, ফ্রেঞ্চ বাষা, স্প্যানিশ বাষা, অনেক জানি'. She did not say “ চিনি' anymore. It suggests that some development has taken place within one year time. She used the word “পরিষ্কার” in several occasions not exactly with its usual meaning. Moreover she used some Korean words like " হাঙ,, হাঙল ত বোলো, সার্ডা .. সার্ডব, গলফু’ directly in her output. It shows that some progression has been taking place. All these, may be, are the traces of Sadia's increasing and progressive knowledge in Bangla morphology. 


\subsection{Syntactic}

Mary's output also shows that her knowledge of Bangla syntax was still in the flux. Her participations were full of grammatical lapses indicating the developing aspect of her understanding of the rules bangla sentence formation. At one stage she said, “ইংলিশ বাষা, আমাকে কঠিন'. Where she could have said, “ইংলিশ বাষা, আমার কাছে কঠিন". Then again, “ইংলিশ বাষা পরিষ্কার করে, পরিক্ষার করে.. চাইনিজ বাষা লন.. লনি ইসে.. সাহা.. বধুসহজ" is not upto Bangla syntax. A little afterward she said, "বাংলা বাষা... রকম দুই মাস কিছু পরিক্কার হয়েছে. হয়েছিলাম" ; first she said "হয়েছে" then she corrects immediately "হরয়েছিाম" to maintain the sequence of tense. A little later she said, "বাংলা ভাষাতে, আমার ভাষা নেই, নেইংড়ঁঁফ আছে, তাই বাংলা ভামাও শিখতে চায়’. It is clear that she was trying to say, “আমাদদর ভাষায় নেই এরকম সাউল্ড বাংলা ভাষায় আছে, তাই..". Then again in the second part of the sentence she uses "ठाয়" with first person. " $চ$ চIয়" is used with second and third person and here “চাই' is appropriate. In Her sentence, “অনেক আদে কোরিয়া, কোরিয়াতে, কোরিয়ায়ে বাষা না হলে, সু স্পিকিং বাষা আছে কিন্ত রাইটিং বাষা নেই, তাহনে.. চাইনিজ থেকে, চাইনিজ লেটার এসেছিলাম, তে চাইনিজ লেটার ব্যবহার করি. অ্যা.. কিন্তু সাউল্ড হইছে’ she failed to maintain the sequence of tense. She wanted to say, "অনেক আগে কারিয়াতে, শ্রু স্পিকিং বাষা ছিল, রাইটিং ভাষা ছিলনা ..”. Same thing happens here again, "ফরটিন সেঞ্চুরিস অ্যাঁ. কোরিায়ান স্কলার এবং কিং মেক ব্যবহার, কোরয়ান ইউনিক বাষা ব্যবহার করেছে'. She used the form “করেছে' whereas, it was proper to say "করেছিল' with fourteenth centuries, a time point in the past. Again here, "আমরা অনেক চাইনিজ লেটার লিখেছে" she used " नিখেছে" inplace of "निখেছিলাম". She said at some point, “আমি ইন্ডিয়াতে.. ইল্ডিয়াতে অনেক চা খেয়েছে.", here as well she failed to use the past form of the verb " খেয়েছিলাম'. At the end of the second interview she said, “... শ্যু ‘ইমাস শিখেছি, শিখেছিলাম'. Though she said "শিখেছি first, but was able to maintain the tense sequence rightly immediately afterward and said, "শিটেছিলামৃ". Another interesting feature of her speech was her use of double plural which is not used in Bangla. She said, "আজকা অনেক মানুষরা ইংলেজি .. সবচেয়ে ভাল শিকেছছ...". It is usually, “আজকাল অনেক মানুষ...”. It shows that within one year time her knowledge of Bangla has improved. We see that Mary's performance in Bangla had lapses of various types like subject-verb agreement, sequence of tense, word order and so on indicating the changing nature of her learning.

\subsection{Summary}

In fine, we see that the features of interlanguage are present in the output of the learners some way or the other in phonetic, morphological and syntactic level. Here we see that all these four learners had interlanguage state to various extents. Lidia was the least successful in acquiring the knowledge of the target language. Kitty's performance was that of a beginner's. Mary exhibits all the features of interlanguage in her effort to communicate in Bangla. She is ready to take risk to convey her message in the TL. Elizabeth's performance suggests that she reached almost a near native proficiency in the TL yet her performance from phonetic consideration clearly shows that she is still stranded in her interlanguage condition. The more one has achieved the accuracy in the target language; the lower is his interlanguage hurdle. The performance of the respondents' can be shown in the following figure where Lidia belongs to the lowest level of the ladder whereas Elizabeth is at the peak:

\section{5- DISCUSSION AND FINDINGS}

\subsection{Analysis}

The interviewee, namely Mary is found to have interlingual state clearly in their performance. Mary displayed all the aspects of interlanguage appear in her output. It goes in favour of the idea of Selinker (chapter two). He opines that it is very hard to overcome interlanguage state phonetically. He cites the example of French and Indian speakers of English who never change no matter how much training is given to them. The major errors of the participant can be shown in a table as follows: (Table in Index 2)

Here we see that the learner displays interlingual identifications in her effort. The deviations that they exhibit comprise all phonetic, morphological and syntactic categories. Her language-learner language can be explained from the standpoints which were upheld by the forerunners of the concept. At this stage we shall try the learner's output by three criteria namely language transfer, overgeneralization, and simplification.

In the output of Mary we find the 'language transfer' feature of interlanguage is at work. "হাঙল, হাঈল ত বোলো, সার্ড .. সার্ডব, গলফু লনি." had been the outcome of direct transfer of the elements of her mother tongue. At another place she said, “খাশি মাংস এবং মাছ মাংস". It can be an example of 'transfer of training' or 'overgeneralization' feature. In Bangla the word “মাংস' is used to indicate different types of meat for example “গবুর মাংস, খাসির মাংস, মুরগীর মাংস”, but in English and in many other languages it is not so. There is a particular word for every kind of meat as in English we have beef, mutton, and chicken and so on. Mary used "মাংস" even for fish overgeneralizing her knowledge of the target language or it might have been caused wrong transfer of training. Same thing might have happened when Mary used " $\overrightarrow{ }$ " suffix 
with “निউজিল্যাল্ডতে" and "बिनि" with "আমि বিভिन्ন বাষাঋৎবহপয, ঝঢ়ধহরংয চিনি, আরো চাইনিজ চিনি'. Bothe for knowing people and language in English 'know' is used. May this is the case with Mary's mother tongue. In Bangla we use "জানি" for knowing a language and "চিনি" for knowing people.

\subsection{Different Factors}

If we try to know the reasons for the variable performances of the learner, we shall see different factors at work. The most obvious one among those factors is L1 interference. Mary's failure to handle the aspirated Bangla sounds may be an example of L1 interference in the phonetic level. She does not have these aspirated sounds in her language namely Korean. She says “বাল লাগে ”ধহফ হড়ঃ্ভাল ...”.

The role of formal instruction is another phenomenon worthy to be considered at this stage. It is generally assumed that the more the instruction, the more is the competence in the target language and lesser is the interlingual state. Mary shows much interlingual identifications in her performance. One understandable reason is she received longer period of instruction. Mary had two months training of Bangla but she has got the prolonged exposure being surrounded by Bangladeshi learner all the time in the hostel and at the institure. She is a good communicator in the target language.

Individual difference factors like motivation may also be at work. In the case of Mary the role of motivation is very obvious. She is strongly motivated; she is to work with TL group of people as language teachers. It may also be the case that Mary has global motivation which aided her a lot.

It is very hard to claim anything cut and dry. Context or language use is of course another influential component to determine the performance of the learners. Complex Socio-culture factors between the learners and the TL group of people might also affecting the performance of the learners in some way or the other which requires further in-depth longitudinal study. To summarise we can enumerate the factors that may work as powerful determinants in the development of interlanguage:

sociological situation

affective factors

amount of exposure - input

opportunities for expression

negative feedback - (note - not correction, but signalling incomprehension)

$>$ absence or presence of pressure on communication

\subsection{Implication for Pedagogy}

Attitude towards learner's error needs be modified. Learners' errors are not something immediately to be eradicated. Rather errors are inevitable and to some extent conducive to language learning. Teachers view, syllabus and teaching materials everything should come in accordance with learners' internal system to let learning take place. It may be said at this stage that the students' errors are a precious resource for the teacher, which inform a teacher about the state of her pupils' interlanguage. It suggests that learning is taking place and the learner is applying various tactics to master the rules of target language. We have to think anew about the traditional negative marking for learners' error. It may affect learning adversely. Interlanguage studies also suggest that there is natural order in acquiring the components of the target language. So, syllabus design should also reflect the learners' preference.

In summing up, we may have some general implications from the study regarding learning Bangla in particular as a second language in respect of the difficulties that the learners face. In the phonetic considerations, it is very much evident that the learner find problems with the aspirated Bangla sounds. They, the aspirated sounds of Bangla, pose a considerable threat for the learners and they cannot handle them accurately. In respect morphology, inflectional and derivational Bangla bound morphemes are also not very easy to deal with. The functional free morphemes like "কিন্তু, অথবা, তাহনে এবং অथठ" are also found to be mixed up by them. Sequence of tense and subject-verb agreement of Bangla syntax also appear to be a tricky area to tackle to the learners.

\subsection{Conclusion}

This research has revealed that the features of interlanguage are very much present in case of the foreigner learner of Bangla studied here and it is helpful to develop a better understanding of the phenomenon in the context of learning Bangla as a second language. The focus of the study here has been the lapses or deviations that the learners make in their effort of learning Bangla and to analyze them from phonetic, morphological and syntactic level. It is surely enlightening to be familiar with all these problems of the learners and at the same time they may offer us an insight in teaching and learning Bangla language in an improved way. Nevertheless, the study of interlanguage is very essential in its own right. It is supposed to give us an access into the intricate learning psychology or the built in syllabus of the learner. According to Richards and Sampson $(1974$, p18) the approximative systems or 
interlanguage (mistakes in traditional sense) are not some harmful pathologies which are only to be removed from the learner, rather they are, may be, the necessary stages in the gradual acquisitions to the target language system. Studying them may lead us to greater understanding of language in general and a more human approach to language teaching. We shall conclude here citing Corder (1967 cited in Richards 1974 p.27) regarding the importance of studying the learners' system. According to him if systematic study of the learners' lapses is made then:

We may begin to be more critical of our cherished notions. We may be able to allow the learner's innate strategies to dictate our practice and determine our syllabus; we may learn to adapt ourselves to his needs rather than impose upon him our preconceptions of how he ought to learn, what he ought to learn when he ought to learn it. (p27, Richards 1974)

Today, finally, we find much of Corder's suggestion is in application in methodology, materials and syllabus design especially in CLT where there are meaningful pragmatic practice, democratic and supportive teacher's role and a changed out look to learners' errors.

\section{ACKNOLEDGEMENT}

I owe gratitude and thanks to several people for the completion of this mini study. First I would like to acknowledge the constant care and guidance of Professor Arifa Rahman. Her valuable suggestions have been a beacon of light all through my research work.

I am also indebted to Professor Waliul Islam and Dr. Sayeedur Rahaman who helped me in various ways. I would like to thank also Professor Mansur Musa and Professor Harunur Rashid who enlightened me with their insights. I must thank two of my colleagues, Mr. Mahmudul Haque, who kindly took the trouble to contact some of my foreign respondents and I am sincerely thankful to Muhammad Shawkat Ali for the use of his Sony IC recorder to collect and preserve data.

Finally, I am grateful to my foreign interviewee who kindly gave their valuable time to undertake my study. She is:

- Ms. Kim So Hyun, a Korean teacher at IML in the Korean Department

Last but not the least, I need to thank Nishat Sharmin, one of my classmates, who gave me her class assignment to consult.

\section{ABOUT THE AUTHOR}

The author has completed his BA (Honors) MA in English from Rajshahi University, Bangladesh in 2000. He has completed his second MA in ELT (English Language Teaching) in 2010 from IML, Dhaka University. He has taught English language and literature in different colleges and universities of Bangladesh till 2012. He is teaching English language skills to Arab EFL learners at King Khalid University since 2012. His articles in English Language and literature are published in different journals home and abroad. He is interested in research, workshops and seminars on Phonetics, sociolinguistics and SLA.

\section{REFERENCES}

[1] Brown, H. D. (1994). Principles of Language Learning and Teaching. Englewood Cliffs: N. J. Prentice Hall Regents.

[2] Corder, S. (1978). "Language -learner language" Understanding Second and Foreign Language Learning ed. Jack C. Richard, Rowley, Mass. : Newbury House. 71-91Corder, S. 1981. Error Analysis and Interlanguage. Oxford: OUP

[3] Ellis, R. (1997). Second Language Acquisition. UK: Oxford University Press

[4] Ellis, R. (1999). Understanding Second Language Acquisition. Oxford: Oxford University Press

[5] Graddol, D. (2006). English Next. UK: The English Company(UK) Ltd. Retrieved from http://www.britishcouncil.org/learning-researchenglish-next.pdf accessed on 20. 9. 2010

[6] Islam, R. (1990). An Introduction to Colloquial Bengali. Dhaka: Bangla Academy

[7] Larsen-Freeman, D. \& Long, M. H. (1991). An Introduction to Second Language Acquisition Research. New York: Longman

[8] Lennenberg, E. H. (1967). Biological Foundations of Language. New York: John Wiley and Sons

[9] Mclaughlin, B. 91987). Theories of SecondLanguage Learning. UK: Edward Arnold

[10] Richards, J. C. (1974). Error Analysis: Perspectives on second Language Acquisition. London: Longman Group, Ltd.

[11] Selinker, L. (1969). "Language Transfer" General Linguistics, 9, p 67 - 92 
[12] Selinker, L. (1972). "Interlanguage" International Review of Applied Linguistics, Vol. $\mathrm{X} / 3,219-231$

[13] Selinker, L. (1992). Rediscovering Interlanguage. London: Longman Group Ltd.

[14] Schmidt, R. (1987) "Sociolinguistic variation and language transfer in phonology" G.Ioup and S. H. Weinberger (eds) Interlanguage Phonology: The acquisition of a second language sound system. New York: Newbury House (pp 365-377) retrieved fromhttp://nflrc.hawaii.edu/PDFs/SCHMIDT\%20Soc iolinguistic \%20variation \%20and\%20language \%20tra nsfer.pdf accessed on 07,09,2011
[15] Weinreich, U. (1953) Language in Contact: Findings and Problems. New York: Publication Circle - 1 